$20-135750$

\title{
GALACTIC NOISE AND PASSIVE MICROWAVE REMOTE SENSING FROM SPACE AT L-BAND
}

\author{
David M. Le Vine and Saji Abraham \\ Goddard Space Flight Center, Greenbelt, MD 20771 \\ dmlevine@priam.gsfc.nasa.gov
}

\begin{abstract}
The spectral window at L-band $(1.4 \mathrm{GHz})$ is important for passive remote sensing of soil moisture and ocean salinity from space, parameters that are needed to understand the hydrologic cycle and ocean circulation. At this frequency, radiation from extraterrestrial (mostly galactic) sources is strong and, unlike the constant cosmic background, this radiation is spatially variable. This paper presents a modern radiometric map of the celestial sky at L-band and a solution for the problem of determining what portion of the sky is seen by a radiometer in orbit. The data for the radiometric map is derived from recent radio astronomy surveys and is presented as equivalent brightness temperature suitable for remote sensing applications. Examples using orbits and antennas representative of those contemplated for remote sensing of soil moisture and sea surface salinity from space are presented to illustrate the signal levels to be expected. Near the galactic plane, the contribution can exceed several Kelvin.
\end{abstract}

\section{INTRODUCTION}

The spectral window 1.400-1.427 GHz (L-band) reserved for passive use only is important for measuring parameters of the Earth surface such as soil moisture and ocean salinity that are needed for understanding the hydrological cycle and energy exchange with the atmosphere. Being able to make observation at the long wavelength end of the microwave spectrum is critical to these measurements. In the case of soil moisture, long wavelengths increase penetration into the soil and mitigate effects of attenuation through the vegetation canopy and the effects of surface roughness. In the case of sea surface salinity, long wavelengths increase the sensitivity to salinity and minimize the dependence on surface temperature and roughness.

However, at L-band radiation from extraterrestrial sources is not negligible. For example, one needs to know the brightness temperature of the celestial sky to correct for down-welling radiation that is reflected from the surface into the receiver $[1,2]$. This contribution is of particular concern for remote sensing of sea surface salinity because the surface is a good reflector and the salinity signal itself is relatively small $[3,4,5]$. The problem is exacerbated by the fact the down-welling radiation is not a constant across the celestial sky, being significantly stronger in the galactic plane $[4,6]$. This is a particular concern in the case of remote sensing from space because the portion of the celestial sky that contributes radiation changes rapidly as the sensor moves through its orbit. In addition, the orbit itself may change its orientation with respect to the celestial sky as is 
the case with sun-synchronous orbits that precess as the Earth rotates around the Sun (to keep the orientation of the orbit with respect to the sun constant).

Previous estimates of the magnitude and distribution of galactic radiation for use in remote sensing $[4,6,7]$ have been rather course. However, recent surveys of the radio sky at $1.4 \mathrm{GHz}[8,9,10,11,12,13]$ have made it possible to produce maps with sufficient spatial and radiometric accuracy to be relevant to remote sensing applications. This paper present a modern map of the radiometric sky at L-band and a solution to the problem of determining the portion of the sky seen by a down-looking radiometer in orbit. The data is presented as equivalent brightness temperature for remote sensing applications. Examples using orbits and antennas representative of those contemplated for remote sensing of soil moisture and sea surface salinity from space are presented to illustrate the signal levels to be expected.

\section{THE RADIO SKY AT L-BAND}

There are three important sources of radiation within the L-band window at 1.413 $\mathrm{GHz}$ that originate outside of our solar system: The cosmic microwave background (CMB), discrete line emission from (mostly) neutral hydrogen and continuum emission such as is emitted by thermal sources. The latter two are the subject of this paper but all three are discussed for completeness.

\section{A. Cosmic Background:}

The cosmic microwave background radiation is a remnant of the origin of the universe in a "big bang". Although the recent cosmological research has focussed on details of its spatial distribution [14], these variations (milli-Kelvin) are not important for applications such as remote sensing of soil moisture or ocean salinity from space. For remote sensing applications, the cosmic background radiation is essentially constant in both space and time with a value of about $2.7 \mathrm{~K}$. This background radiation can contribute to a measurement with a down-looking radiometer in a direct manner if, for example, the radiometer antenna has side lobes above the horizon. It also can contribute via reflection of down-welling radiation off the surface [1]. The latter is especially important in remote sensing of the ocean surface where the reflection coefficient is relatively large. The cosmic background will be included in the examples to be presented here; but, since it is uniform and constant over the spectral window, it is relatively easily included in radiometer retrieval algorithms $[1,7]$.

\section{B. Line Emission:}

The window at $1.413 \mathrm{GHz}$ was protected for passive use because of the interest in emission from a hyperfine transition in neutral hydrogen that occurs in this window. The original proposal that such radiation could be detected from neutral hydrogen in our galaxy is attributed to Oort and van de Hulst, and the first observations were made by Ewen and Purcell in 1951 [12]. This radiation provides information on the temperature, density and motion of hydrogen. The radiation is concentrated around the plane of the 
galaxy, but clouds of hydrogen are widespread and no direction is observed without some such radiation.

Several surveys of this source of radiation have been made $[15,16,17,18,19$, 20]. Recently, Hartman and Burton [12] motivated by the high quality, all-sky surveys being made at other wavelengths, reported a new survey. The Leiden/Dwingeloo survey (Table I) used a 25-meter radio telescope and covered the sky above declination of $-30^{\circ}$. This survey was recently complemented by data collected with the 30 meter dish antenna at the Instituto Argentino de Radioastronomia (IAR) and reported by Arnal et al [13]. The IAR survey (Table I) covered declinations south of -25 degrees filling in the missing portions of the southern sky. The result is data with sufficient spatial resolution $\left(0.5^{\circ} \mathrm{x}\right.$ $\left.0.5^{\circ}\right)$ and radiometric resolution $(\Delta T<0.1 \mathrm{~K})$ to be applicable to remote sensing at $\mathrm{L}$ band from space, including high-resolution sensors proposed for the future (e.g. a spatial resolution of $0.5^{\circ} \times 0.5^{\circ}$ corresponds to an aperture of about 25 meters).

\section{Continuum Radiation}

In addition to the discrete spectra associated with atomic transitions as in hydrogen above, there is a continuum of radiation from extra-terrestrial sources. This radiation can be divided into thermal and non-thermal sources. Thermal sources have a spectrum (amplitude versus frequency) similar to that of blackbody radiation. At L-band, the Rayleigh-Jeans law applies and the power increases with frequency as $\mathrm{f}^{2}$. Nonthermal sources are sources whose spectra behave differently. An example is synchrotron radiation from relativistic electrons, in which case the spectrum at L-band decreases with frequency roughly as $f^{-0.75}$ [21]. The source of continuum radiation may be localized in space (discrete sources) or may be of a spatially continuous nature (diffuse or unresolved discrete sources). The source of the radiation is mostly galactic because these sources are closest, but there are also strong extra-galactic sources such as the "radio galaxy" Cygnus A [21].

There have been several surveys of the continuum radiation at $1.4 \mathrm{GHz}[22,23$, $24,25,26,27]$. None of these surveys covered the entire sky and they use different bandwidths and the measurements were made with differing sensitivity. For the most part, they consist of a map of discrete sources. However, for applications to remote sensing of the Earth, it is desirable to have the integrated signal from a particular direction in the sky (i.e. power from all sources, discrete and continuous), because antennas likely to be employed in space in the foreseeable future will not resolve individual sources. The recent survey with the Stockert telescope at Bonn University [8], [9] provides data in this format. The antenna had a half-power beam width of about 0.5 degrees. The sensitivity of these measurements is about $0.05 \mathrm{~K}$ and the absolute calibration (zero level accuracy) is $0.5 \mathrm{~K}$. This survey (Stockert survey; Table I) covers all of the northern sky and the southern sky to $-19^{\circ}$ declination. The data includes all sources except for a region around Cassiopia A which was too strong. Recently, the survey of the southern sky was completed using the $30-\mathrm{m}$ radio telescope of the Instituto Argentino de Radioastronomia [10], [11]. The IAR continuum survey (Table I) covers the 
southern sky for declination below $-10^{\circ}$ with spatial resolution and sensitivity similar to that of the Stockert survey.

\section{DATA in Remote Sensing Format}

It is common practice in passive microwave remote sensing to treat the scenes as thermal sources and the receivers as narrow bandwidth devices [7,21]. In the RayleighJeans limit, this permits one to describe the measurements and scenes in terms of an equivalent "brightness" temperature. For sources that are not blackbodies, the brightness temperature is the product of the physical temperature and emissivity of the surface. The emissivity is a function of the properties of the surface, hopefully including the parameters of interest. This is the approach proposed to measure soil moisture and sea surface salinity $[4,28,29]$ in which case water and salt change the emissivity sufficiently that the resulting changes in brightness temperature can be detected.

In the sections below, the data from the radio astronomy surveys will be presented in the form of an equivalent blackbody temperature. The data will be presented as an equivalent thermal source normalized such that total power is $P=k T_{B} \Delta B$. For this purpose, a bandwidth, $\Delta \mathrm{B}=20 \mathrm{MHz}$, has been assumed. This represents a reasonable maximum for the window at $1.413 \mathrm{GHz}$ after allowances for filter shape.

\section{A. Line Emission}

The line emission has a relatively narrow spectrum [21]. For hydrogen at rest, it occurs at the frequency associated with the hyperfine transition at $21.106 \mathrm{~cm}$. However, the line is shifted by motion of hydrogen relative to the observer (doppler shift) and spread by thermal energy of the gas (collisions and vibrations). But, even with large Doppler shift and thermal broadening, the spectrum of this radiation is relatively narrow. For example, the Leiden/Dwingeloo survey [12] and IAR survey [13] cover the velocity range from -450 to $+400 \mathrm{~km} / \mathrm{s}$ (Table I) which corresponds to a frequency range of less than $\pm 2.2 \mathrm{MHz}$ about the center (at rest) frequency of $1.42 \mathrm{GHz}$. The two surveys both report power integrated over the spectrum of the line. The integrated power is given in $\mathrm{K}$ $\mathrm{km} / \mathrm{s}$.

To convert into a format useful for remote sensing, this data was first converted to $\mathrm{K}-\mathrm{MHz}$ using the standard form for Doppler shift: $v=v_{0}(1-v / \mathrm{c})$ where $v$ is velocity. Then, this value was divided by $20 \mathrm{MHz}$ to convert it into an effective brightness temperature. The brightness temperature, $T_{B}$, is the temperature of a blackbody that observed with an ideal receiver with a bandwidth of $\Delta B=20 \mathrm{MHz}$, will give the power

$\left(\mathrm{P}=\mathrm{kT} \mathrm{T}_{\mathrm{B}} \Delta \mathrm{B}\right)$ reported in the radio astronomy surveys. The data can be converted for use with receivers with other bandwidth with the obvious re-normalization. (Of course, this only makes sense if the receiver bandwidth is centered on the line and is greater than \pm 2.2 $\mathrm{MHz}$.)

The data in the above form is shown in Figure 1 (top). The units are Kelvin and the plot is in celestial coordinates. The " $U$ ' shape region of high brightness temperature 
is the plane of the galaxy (which is tilted with respect to an observer in the celestial coordinate system). The effective brightness temperature is small almost everywhere except in the galactic plane where equivalent blackbody temperatures on the order of $3 \mathrm{~K}$ can occur.

\section{B. Continuum Radiation}

For the continuum radiation, the data used here was the Stockert survey [9] for the northern sky together with the more recent IAR survey [10] for the southern sky (Table I). In both cases, the data are in the form of total power over the bandwidth of the receiver with the exception that the line emission from hydrogen was excluded (removed with a narrow filter at the line center). The power represents radiation from all sources within the beam, discrete and unresolved and includes thermal and non-thermal sources. However, Cassiopeia A, which was too strong to be included in the Stockert survey, and a small region of the sky around Cassiopeia $A$ is excluded from the data.

For both surveys, the data are in the form of power integrated over the pass-band of the radiometer receiver. The effective bandwidth was about $18 \mathrm{MHz}$ and $13 \mathrm{MHz}$ for the Stockert and Villa Elisa surveys, respectively (after correction for the filter to remove line emission). The Villa Ellisa antenna $(30 \mathrm{~m})$ was under-illuminated to match the resolution of $25 \mathrm{~m}$ antenna used in the Stockert survey [10, 11].

The data from the Stockert survey [9] normalized to a bandwidth of $20 \mathrm{MHz}$ is shown in celestial coordinates in bottom panel of Figure 1. (All of data in Figure 1 has been converted to celestial coordinates using the J2000 epoch [21, 32]). As in the case of

the line emission from hydrogen, the strongest radiation tends to lie along the plane of the galaxy as is evident on the far right hand side. Also notice the white spot at the upper right (Declination $60^{\circ}$ and Right Ascension $355^{\circ}$ ) which is the data missing around Cassiopeia A. Data exists for the portion of the southern sky missing in Figure 1. However, this data (IAR continuum survey; Reich, 2001) has not been released at the resolution shown in Figure 1. The data has been provided for use in this paper at lower resolution [30] and is presented and discussed in Sections IV-V.

\section{Examples}

Figure 2 shows examples of the magnitude and spatial distribution of the data in more detail. The data shown are cuts through the color-coded maps in Figure 1 at fixed declination of $0^{\circ}, 20^{\circ}$ and $40^{\circ}$. On the left side of Figure 2 is shown the effective brightness temperature due to line emission from hydrogen (from Figure 1; top) as a function of right ascension and on the right side is shown the effective brightness temperature for the continuum radiation (from Figure 1; bottom). The peaks in these curves are associated with crossing the galactic plane. Notice that the contribution from the continuum is considerably larger than from the line emission from hydrogen. In particular, peak values of effective brightness temperature from line emission are on the order of $2 \mathrm{~K}$ whereas the peak value due to continuum radiation is nearly $20 \mathrm{~K}$. Also, 
notice that the distribution is quite complex with the obvious peak at the galactic plane but with levels that depend on where the intersections with the galactic plane occur.

\section{Effect of Antenna Beam}

The apparent brightness temperature actually seen in a remote sensing application will depend on the antenna employed. The antenna smoothes (integrates) the incident radiation and, as a result, the observed value can be significantly different from the peak values in Figure 1. This is especially true in the vicinity of the galactic plane, which is relatively narrow.

In order to understand the effect of the antenna, one could integrate over the portion of the celestial sky contributing to the measurement. That is, take the convolution with the power pattern of the antenna (e.g. Section 3.4 of [21]). However, it is equivalent to perform the convolution over the entire sky first and then locate the portion of the sky contributing to the measurement. This approach has the advantage of presenting the smoothed data in its entirety independent of the particular application. This has been done here.

The data is presented in Figure 3 for an antenna with a Gaussian beam with a full width at half maximum (FWHM) of $10^{\circ}$. The details of the integration are presented in Appendix A. The choice of $10^{\circ}$ beam width was made to indicate the effect of smoothing but to remain conservative and not overly smooth the data for remote sensing applications. Figure 3 (top) shows the smoothed data for line emission and Figure 3 (bottom) is the smoothed data for the continuum radiation. In the case of the continuum radiation, the data from the Stockert survey (Table I) was smoothed as outlined in Appendix A and the data for the southern sky (IAR continuum survey) was provided to us with the 10 degree smoothing (courtesy of P. Reich [30]). Notice that the general features of the high-resolution maps remain in the smoothed data.

Figure 4 shows detail for cuts through the data at fixed declination of $0^{\circ}, 20^{\circ}$ and $40^{\circ}$. The smoothed line emission is shown on the left and the continuum radiation is on the right. Notice that the general features evident in the high-resolution data (Figure 2) remain, although the large difference in the peak value between line emission and continuum at 40 degrees is much reduced. This reflects the very narrow nature of the peak in the continuum radiation on the galactic plane. Also, notice that away from the galactic plane, the continuum radiation $(\sim 1 \mathrm{~K})$ is greater than from line emission $(\sim 0.05$ K).

Figure 5 is an example at 20 degrees declination of the total from the three contributions: Cosmic background, line emission and continuum. Shown at the top is the original and smoothed data for the line emission. In the middle is the original and smoothed data for the continuum radiation. The bottom panel shows each of the components (smoothed line emission, smoothed continuum and the cosmic background) together with the sum. Notice that the plane of the galaxy is evident and that the contribution of the continuum and line emission is clearly important and comparable to 
the CMB. The peak around the galactic plane depends on declination and, for example, would be much larger if a declination of 40 degrees were shown (see Figures 2 and 4 ).

It is clear from Figures 1-5 that the background radiation is spatially complex. Large values are possible along the galactic plane. On the other hand, there are regions near the galactic pole where the line emission and continuum radiation are small $(\leq 1 \mathrm{~K})$ and the cosmic background dominates. Clearly, it is important to know what portion of the "sky" is contributing to a particular measurement. This problem is addressed in Section V.

\section{REMOTE SENSING PROBLEM}

The data from the radio astronomy surveys indicates that the background radiation can be significant and highly variable. Consequently, for remote sensing applications it is important to know what portion of the celestial sky is contributing to a particular measurement. To address this problem, imagine a radiometer at L-band in orbit circling the Earth with its antenna pointing down. The goal is to determine the contribution from the radio sky (down-welling radiation that is reflected into the antenna), which must be taken into account at each position in this orbit. The input is the data given in Figure 1 (or Figure 3 ) normalized to the radiometer bandwidth, plus a constant of $2.7 \mathrm{~K}$ that is added to account for the cosmic microwave background.

The solution can be found by tracing rays from the antenna to the surface and computing how they are reflected toward the sky. For example, a flat, specular surface behaves like a mirror and the solution can be obtained by placing the antenna at its conjugate point behind the surface and looking out in the direction of the specularly reflected ray. The antenna pattern is unchanged, but it does undergo a mirror image change of symmetry (i.e. right hand symmetry becomes left hand). One computes the power reflected into the antenna by integrating the mirrored antenna pattern over the sky.

One can follow a similar procedure to solve the problem for a curved earth. The solution is outlined in Appendix B and solved for an ideal case (spherical earth, circular orbit and specular surface). Examples are shown in Figure 6 for the case of an antenna that looks to the side in a plane perpendicular to the orbit (e.g. as in a cross track scan). At the left are the smoothed data describing the radio sky at L-band in celestial coordinates (sum of line emission and continuum) seen by the antenna (Gaussian power pattern with a 10 degree beam width). Shown on the smoothed data is the locus traced by the reflected ray at bore-sight for an antenna looking to the right at $\theta_{\mathrm{i}}=30$ degrees in a circular orbit with inclination angle is 95 degrees (angle $\gamma$ in Equations 4B). It is possible to plot orbits on the original data and then integrate over the antenna beam. However, as mentioned above, it is more efficient to integrate first and then plot the orbits. This what has been done in creating Figure 6. The panels on the right show the brightness temperature along this locus as a function of declination. Four values are plotted: The line emission, continuum emission, CMB (the straight line) and the total. 
The shape of the loci in Figure 6 depends on the incidence angle of the antenna and the inclination of the orbit. The position of the locus (i.e. center of the curve) is determined by the intersection of the plane of the orbit with the plane of the equator. This is illustrated by the two examples shown in Figure 6 that differ only in that the intersection of the plane of the orbit has been rotated by 90 degrees. These two examples show that keeping track of changes in the orbit, for example due to precession, can be important for determining the background radiation. This is illustrated further in the section below using the orbit for a mission proposed several years ago to measure soil moisture from space. The problem of relating the orbit as seen in an earth-centered coordinate system (e.g. equatorial intersection as a function of longitude and local time) to celestial coordinates is discussed in Appendix $\mathrm{C}$.

\section{The HYDOSTAR Orbit}

An example is presented here for a sun-synchronous orbit with a $6 \mathrm{am} / 6 \mathrm{pm}$ equatorial crossing. This is an orbit commonly selected for remote sensing of soil moisture [28], [31] and was the orbit for a sensor proposed to measure soil moisture from space called HYDROSTAR [28]. A sun-synchronous orbit is one oriented such that all observers on the Earth sees the satellite pass overhead at the same local time. It is the orbit of choice for many remote sensing applications because the local time of observation is constant. In practice, this orbit generally has a high inclination (passes close to the pole) and the equatorial crossing time precesses a bit about the nominal value.

In order to remain in the same local orientation with respect to the sun, the orbit must precess in celestial coordinates as the earth rotates about the sun. The change is about 1 degree per day. As a result of the precession, the position of the locus of the reflected rays in celestial coordinates (Figure 6) will drift across the sky going through a change of 360 degrees in right ascension each year. The problem of locating the orbit in celestial coordinates given the local time of equatorial crossing is the problem in astronomy of transforming local time into sidereal time. The solution used here is given in Appendix $\mathrm{C}$. Once the orbit is located in celestial coordinates, the procedure outlined in Section $\mathrm{V}$ above is followed to plot the locus of the reflected rays on the sky. (This amounts to neglecting changes of the orbital plane during one period, about 90 minutes.)

Figure 7 shows examples with the HYDROSTAR orbit for several times of the year starting with March 15,2002. The calculation is for the antenna described above ( $10^{\circ}$ Gaussian beam) looking right at 5 degrees $\left(\theta_{i}=5\right)$. The orbit inclination is 95 degrees. The curves on the left show the orbit in celestial coordinates. The curves on the right show the total effective background radiation, the sum of the three terms, line emission, continuum and $\mathrm{CMB}$. Notice that the total varies from a little less than $4 \mathrm{~K}$ to nearly $11 \mathrm{~K}$. The values change over the orbit (i.e. in one period). Also, the distribution over the orbit varies seasonally (i.e. with the time of year). The values would also change if the incidence angle, $\theta_{i}$, where changed. 


\section{CONCLUSION}

In addition to the uniform cosmic background radiation (CMB), there is additional radiation (line emission from hydrogen and a continuum background) that must be taken into account for remote sensing at L-band. In contrast to the $\mathrm{CMB}$, this additional radiation is spatially varying and strongest in the direction of the plane of the galaxy. The effective brightness temperature of down-welling radiation from these sources that is reflected from the surface into the radiometer depends on the antenna beam width and surface conditions. For a perfectly reflecting surface (reflectivity of unity) and an antenna with a beam width on the order of 10 degrees, the peak contribution from sources other than the $\mathrm{CMB}$ is on the order of $1-6 \mathrm{~K}$, varying with the orientation of the sensor and orbit. The fact that this signal can change both seasonally and with the location of the sensor in its orbit makes its presence an important issue for remote sensing of the Earth. The importance of the background radiation depends on the applications and surface conditions. For example, it is less an issue for applications such as the remote sensing of soil moisture where the signal is large and the reflectivity at the surface small (on the order of 0.3). However, it is more important for remote sensing applications such as the measurement of sea surface salinity where the reflectivity of the surface is large (on the order of 0.7 ) and the signal is small (on the order of $0.5 \mathrm{~K}$ per psu). In the latter case, careful mapping of the down-welling radiation will be an important issue.

\section{REFERENCES}

[1] C. T. Swift, "Passive microwave remote sensing of the ocean- A review," Bound. Layer Meteorol., vol. 18, pp. 25-54, 1980.

[2] H-J. C. Blume and B. M. Kendall, "Passive microwave measurements of temperature and salinity in coastal zones," IEEE Trans. Geosci. Remote Sensing, vol. GE-20, pp. 394-404, 1982.

[3] H-J. C. Blume, B. M. Kendall and J. C. Fedors, "Measurement of ocean temperature and salinity via microwave radiometry," Boundary-Layer Meteorol., vol. 13, pp. 295308, 1978.

[4] C. T. Swift and R. E. McINTOSH, "Considerations for Microwave remote sensing of ocean-surface salinity," IEEE Trans. Geosci. Remote Sensing, vol. GE-21, No. 4, 480-491, 1983.

[5] E. G. Njoku, W. J. Wilson, S. H. Yueh, and Y. Rahmat-Samii, "A large-antenna microwave radiometer-scatterometer concept for ocean salinity and soil moisture sensing," IEEE Trans. Geosci. Remote Sensing, vol. 38, pp. 480-491, 2000.

[6] H. C. Ko, "The distribution of cosmic radio background radiation," Proc. IRE, vol. 46, pp. 208-215, 1958. 
[7] F. T. Ulaby, R. K. Moore and A. K. Fung, Microwave remote sensing fundamentals and radiometry, Microwave remote sensing: Active and Passive, vol. 1. AddisonWesley publishing company, 1981.

[8] W. Reich, "A radio continuum survey of the northern sky at $1420 \mathrm{MHz}$ - Part 1," Astronomy and Astrophysics supplement series, vol. 48, pp. 219-297, 1982.

[9] P. Reich and W. Reich, "A radio continuum survey of the northern sky at $1420 \mathrm{MHz}$ - Part II,” Astronomy and Astrophysics supplement series, vol. 63, pp. 205-292, 1986.

[10] P. Reich, J. C. Testori and W. Reich, "A radio continuum survey of the southern sky at $1420 \mathrm{MHz}$, The atlas of contour maps," Astronomy \& Astrophysics, vol. 376, pp. 861-877, 2001.

[11] J. C. Testori, P. Reich, J. A. Bava, F. R. Colomb, E. E. Hurrel, J. J. Larrarte, W. Reich and A. J. Sanz, "A radio continuum survey of the southern sky at $1420 \mathrm{MHz}$ : Observations and data reduction," Astronomy and Astrophysics, vol. 368, pp. 1123 $1132,2001$.

[12] D. Hartmann and W. B. Burton, Atlas of Galactic Neutral Hydrogen. Cambridge Univ. Press, 1997.

[13] E. M. Arnal, E. Bajaja, Larrarte, R Morras and W G L Pöppel, "A high sensitivity HI survey of the sky at $\delta \leq-25^{\circ}$," Astronomy and Astrophysics Supplement Series, vol. 142 , pp. $35-40,2000$.

[14] J. C. Mather, E. S. Cheng, R. E. Eplee, Jr., R. B. Isaacman, S. S. Meyer, R. A. Shafer, R. Weiss, E. L. Wright, C. L. Bennett, N. W. Boggess, E. Dwek, S. Gulkis, M. G. Hauser, M. Janssen, T. Kelsall, P. M. Lubin, S. H. Moseley, Jr., T. L. Murdock, R. F. Silverberg, G. F. Smoot and D. T. Wilkinson, "A preliminary measurement of the cosmic microwave background spectrum by the Cosmic Background Explorer (COBE) satellite," The Astrophysical Journal, vol. 354, pp. L37-LA0, 1990.

[15] M. N. Cleary, C. Heiles and C. G. T. Haslam, "A synoptic view of the galaxy in HI," Astronomy and Astrophysics Supplement Series, vol. 36, pp. 95-127, 1979.

[16] F. R. Colomb, W. G. L. Poppel and C. Heiles, "Galactic HI at $\mid \mathrm{bl} \geq 10^{\circ}$. II. Photographic presentation of the combined southern and northern data," Astronomy and Astrophysics Supplement Series, vol. 40, pp. 47-55, 1980.

[17] G. Westerhout, G. L. Mader and R. H. Harten, "Telescope beam characteristics and temperature scale of the Maryland-Green Bank 21-cm line survey," Astronomy and Astrophysics Supplement series, vol. 49, pp. 137-141, 1982. 
[18] F. J. Kerr, P. F. Bowers, P. D. Jackson and M. Kerr, "Fully sampled neutral hydrogen survey of the southern milky way," Astronomy and Astrophysics supplement series, vol. 66, pp. 373-504, 1986.

[19] W. B. Burton, Galactic and Extragalactic Radio Astronomy. (G. L. Verschuur and K. I. Kellerman, eds.), Springer-Verlag, New York, 1988.

[20] W. B. Burton, The Galactic Interstellar Medium. (D. Pfenniger and P. Bartholdi, eds.), Springer-Verlag, Heidelberg, 1992.

[21] J. J. Condon and J. J. Broderick, "A $1400 \mathrm{MHz}$ sky survey. 1. Confusion-limited maps covering $7^{\mathrm{h}} 30^{\mathrm{m}}<\alpha<19^{\mathrm{h}} 30^{\mathrm{m}},-5^{\circ}<\delta<+82^{\circ}$," The Astronomical Journal, vol. 90 , p. $2540,1985$.

[22] J. D. Kraus, Radio Astronomy. McGraw-Hill Inc, 1966.

[23] R. S. Dixon, “A master list of radio sources," Astrophys. J. Suppl. Ser, vol 20, No. $180,1970$.

[24] A. E. Wright and R. Otrupcek, Parkes Radio Sources Catalogue, Version 1.01. Australia Telescope National Facility, 1990.

[25] W. Reich, P. Reich and E. Fürst, "The Effelsberg $21 \mathrm{~cm}$ radio continuum survey of the Galactic plane between $\mathrm{l}=357^{\circ}$ and $\mathrm{l}=95.5^{\circ}$," Astronomy and Astrophysics Supplement Series, vol. 83, pp. 539-568, 1990.

[26] R. L. White and R. H. Becker, "A new Catalog of 30,239 $1.4 \mathrm{GHz}$ sources," The Astrophysical Journal Supplement Series, vol. 79, pp. 331-467, 1992.

[27] B. Uyaniker, E. Fürst, W. Reich, P. Reich, and R. Wielebinski, "A 1.4 GHz radio continuum and polarization survey at medium Galactic latitudes," Astronomy \& Astrophysics Supplement Series, vol. 138, pp. 31-45, 1999.

[28] D. M. Le Vine, J. B. Zaitzeff, E. J. D'Sa, J. L. Miller, C. Swift, M. Goodberlet, “Sea surface salinity: Toward an operational remote-sensing system," in Satellites, Oceanography and Society. (D. Halpern, Ed.), pp. 321-335, Elsevier Oceanography Series \#63, Elsevier Science, 2000.

[29] P. Waldteufel, E. Anterrieu, J. M. Goutoule and Y. Kerr, "Field of view characteristics of a 2-D interferometric antenna, as illustrated by the MIRAS/SMOS L-band concept," in Microwave Radiometry and Remote Sensing of the Earth's Surface and Atmosphere. (Pampaloni P. and S. Paloscia, Eds.), VSP, The Netherlands, pp. 467-475, 2000.

[30] P. Reich, Private Communication, 2002. 
[31] Y. H. Kerr, P. Waldteufel, J.-P. Wigneron, J. M. Martinuzzi, B. Lazard, J.-M. Goutoule, C. Tabard and A. Lannes, "The soil moisture and ocean salinity mission: an overview," in Microwave Radiometry and Remote Sensing of the Earth's Surface and Atmosphere. (Pampaloni P. and S. Paloscia, Eds.), VSP, The Netherlands, pp. 467-475, 2000.

[32] D. A. Vallado and W. D. McClain, Fundamentals of Astrodynamics and Applications. McGraw.Hill, 1997.

Table I: Summary of the survey parameters

\begin{tabular}{|l|c|c|c|c|}
\hline \multicolumn{1}{|c|}{ Parameters } & $\begin{array}{c}\text { Stockert } \\
\text { Continuum } \\
\text { Survey }\end{array}$ & $\begin{array}{c}\text { IAR } \\
\text { Continum } \\
\text { Survey }\end{array}$ & $\begin{array}{c}\text { Leiden/Dwingeloo } \\
\text { Hydrogen Survey }\end{array}$ & $\begin{array}{c}\text { IAR } \\
\text { Hydrogen Survey }\end{array}$ \\
\hline Coverage & $\begin{array}{c}0^{\circ} \leq \leq 360^{\circ} \\
\delta \geq-19^{\circ}\end{array}$ & $\begin{array}{c}0^{\circ} \leq \alpha \leq 360^{\circ} \\
\delta \leq-10^{\circ}\end{array}$ & $\begin{array}{c}0^{\circ} \leq \alpha \leq 360^{\circ} \\
\delta \geq-30^{\circ}\end{array}$ & $\begin{array}{c}0^{\circ} \leq \alpha \leq 360^{\circ} \\
\delta \leq-25^{\circ}\end{array}$ \\
\hline HPBW (effective) & $\sim 35^{\circ}$ & $35^{\prime} .4$ & $36^{\circ}$ & $30^{\circ}$ \\
\hline $\begin{array}{l}\text { Effective } \\
\text { sensitivity }\end{array}$ & $50 \mathrm{mK}$ & $\sim 50 \mathrm{mK}$ & $70 \mathrm{mK}$ & $70 \mathrm{mK}$ \\
\hline Velocity range & $\mathrm{N} / \mathrm{A}$ & $\mathrm{N} / \mathrm{A}$ & $-450 \leq \mathrm{vlsr} \leq 400 \mathrm{~km} / \mathrm{s}$ & $-450 \leq \mathrm{vlsr} \leq 400 \mathrm{~km} / \mathrm{s}$ \\
\hline Velocity resolution & $\mathrm{N} / \mathrm{A}$ & $\mathrm{N} / \mathrm{A}$ & $1.03 \mathrm{~km} / \mathrm{s}$ & $1.27 \mathrm{~km} / \mathrm{s}$ \\
\hline $\begin{array}{l}\text { Effective } \\
\text { bandwidth }\end{array}$ & $18 \mathrm{MHz}$ & $13 \mathrm{MHz}$ & & $30 \mathrm{~m}$ \\
\hline Antenna diameter & $25 \mathrm{~m}$ & $30 \mathrm{~m}$ & $25 \mathrm{~m}$ & \\
\hline
\end{tabular}

Notes: $\alpha$ is right ascension and $\delta$ is declination. 


\section{APPENDIX A \\ Integration Over The Antenna Beam}

The objective is to compute the effective brightness temperature, $T_{B}$, of the background radiation when observed with an antenna representative of those one might use for remote sensing from space. In particular, if $P_{n}(\theta)$ is the normalized power pattern of the antenna, it is desired to compute:

$$
\mathrm{T}_{\mathrm{B}}\left(\Omega_{\mathrm{o}}\right) \quad=\left(1 / \Omega_{\mathrm{a}}\right) \int \mathrm{T}(\Omega) \mathrm{P}_{\mathrm{n}}\left(\Omega_{\mathrm{o}}-\Omega\right) \sin (\theta) \mathrm{d} \varphi \mathrm{d} \theta
$$

Where, $\Omega$ denotes a direction (point in the celestial sky) with spherical coordinates $(\varphi, \theta)$, $\mathrm{d} \Omega=\sin (\theta) \mathrm{d} \varphi \mathrm{d} \theta$ and $\Omega_{\mathrm{a}}$ is the beam solid angle:

$$
\Omega_{\mathrm{a}} \quad=\int \mathrm{P}_{\mathrm{n}}(\Omega) \mathrm{d} \Omega
$$

The calculations will be done here for an antenna with a Gaussian power pattern with full width at half-maximum (FWHM) of $\theta_{\mathrm{b}}$ :

$$
P_{n}(\theta)=\exp \left\{-\alpha\left(2 \theta / \theta_{b}\right)^{2}\right\}
$$

where $\alpha=\ln (2)=0.6931$.

Since the data is presented at discrete points in celestial coordinates, this suggests doing the integration (Equation 1A) in celestial coordinates: $\alpha=\varphi ; \delta=\pi / 2-\theta$. One obtains:

$$
\mathrm{T}_{\mathrm{B}}\left(\alpha_{\mathrm{o}}, \delta_{\mathrm{o}}\right)=\left(1 / \Omega_{\mathrm{a}}\right) \sum \mathrm{T}\left(\alpha_{\mathrm{i}}, \delta_{\mathrm{i}}\right) \mathrm{P}_{\mathrm{n}}\left(\Theta_{\mathrm{i}}\right) \cos \left(\delta_{\mathrm{i}}\right) \Delta \alpha \Delta \delta
$$

The sum is over all data points within the beam. The major issue is to determine the angle $\Theta_{i}$ from antenna boresight at $\left(\alpha_{0}, \delta_{0}\right)$ to the data at point $\left(\alpha_{i}, \delta_{i}\right)$. The angle needed is the polar angle in spherical coordinates (Equation 3A). This angle can be found by rotating coordinates from the original system ( $\mathrm{z}$-axis toward the north pole) into one with the $\mathrm{z}$-axis aligned with the antenna boresight. (See Appendix B.) One obtains:

$$
\cos \left(\Theta_{i}\right)=\cos \left(\delta_{0}\right) \cos \left(\delta_{i}\right) \cos \left(\alpha_{0}-\alpha_{i}\right)+\sin \left(\delta_{0}\right) \sin \left(\delta_{i}\right)
$$

The calculations in the text (Figure 3) have been carried out for the case $\theta_{b}=10^{\circ}$. This was done with a simple search in which each $\Theta_{i}$ is computed for each point $\left(\alpha_{i}, \delta_{i}\right)$. The sum should be over all space, but the antenna beam decreases very quickly and it was found that an integration out to about $\Theta_{\mathrm{i}} \cong 1.5 \theta_{\mathrm{b}}$ was adequate. Also, to reduce the computational time, the antenna bore sight $\left(\alpha_{0}, \delta_{0}\right)$ was stepped across the map in $1^{\circ} \times 1^{\circ}$ increments. 


\section{Appendix B}

\section{Location of Antenna Bore Sight on Celestial Sky}

Imagine a satellite in orbit about Earth. Assume that the plane of the orbit is fixed and independent of rotation of Earth about its axis. Also imagine a sensor on this satellite with an antenna that looks down toward the Earth surface. It is desired to determine the radiation from the celestial sky that is reflected from the surface into the antenna. The objective of this appendix is to determine where on the celestial sphere this radiation originates and to trace the locus of this spot as the satellite rotates in its orbit.

The solution is found by, tracing rays from the antenna to the surface and computing how they are reflected toward the sky. To solve the problem, make the following assumptions:

1. The Earth is spherical;

2. The orbit is circular;

3. The Earth surface is specular.

In actuality the power received by the radiometer will depend on the properties of the surface (roughness, dielectric constant, etc.), but since these are specific to each application, they are being ignored here. The ideal case will be an upper bound.

First consider the case when the antenna looks across track (i.e. perpendicular to the orbit) at an angle $\theta_{\mathrm{i}}$. The geometry is given in Figure $1 \mathrm{~B}$ where $\mathrm{R}_{\mathrm{e}}$ is the radius of the Earth and $\mathrm{h}$ is the altitude of the satellite above the surface. The specular angle is $\theta_{\mathrm{s}}$ and is given by:

$$
\theta_{\mathrm{s}}=\sin ^{-1}\left\{\left[\left(\mathrm{R}_{\mathrm{e}}+\mathrm{h}\right) / \mathrm{R}_{\mathrm{e}}\right] \sin \left(\theta_{\mathrm{i}}\right)\right\}
$$

Obviously there is an upper limit, $\theta_{\mathrm{l}}=\sin ^{-1}\left(\mathrm{R}_{\mathrm{e}} /\left(\mathrm{R}_{\mathrm{e}}+\mathrm{h}\right)\right)$, above which rays no longer encounter the Earth. For larger angles, the antenna receives radiation directly from the sky in the direction of the antenna beam. However, when this limit is not exceeded, the radiation incident on the antenna comes from the direction of the specularly reflected rays. Since, the celestial sources are very far away, these rays can be assumed to come from the center of the Earth (or Sun) with negligible error. The curvature of the Earth will cause slight divergence of the rays and a mirror-image change in rotational symmetry. To a first approximation, the divergence will be neglected. Also, only antennas with rotationally symmetric antenna patterns, for which the change in symmetry is unimportant, will be considered. With these assumptions, the radiation can be calculated by translating the antenna to the center of the Earth and imagining that it looks out in the direction of the reflected ray (i.e. $\theta_{s}$ ).

Now consider the motion of the satellite in orbit. The plane of the orbit is defined by its normal, the vector $\mathrm{ON}$ in Figure $1 \mathrm{~B}$, using the right hand rule with respect to the direction of rotation of the satellite. As the satellite rotates, the ray in the direction $\theta_{s}$ traces a cone with interior angle, $\Omega$, with respect to the normal: 


$$
\Omega \quad=90-2 \theta_{s}+\theta_{j} .
$$

The last step is to plot this ray in celestial coordinates. This is done in the following steps. First imagine an earth-centered coordinate system with the $\mathrm{z}$-axis in the direction of the north pole and the $x$-axis pointing in the direction of the vernal equinox (zero right ascension). Standard spherical coordinates $(\theta, \varphi)$ are related to celestial coordinates "declination", $\delta$, and "right ascension", $\alpha$, as follows:

$$
\begin{aligned}
& \alpha=\varphi \\
& \delta=90-\theta
\end{aligned}
$$

Now, rotate coordinates to a new system with the $\mathrm{z}^{\prime}$-axis in the direction of the normal to the orbit. Rotate as follows:

1. About the $z$-axis through an angle, $\psi$

2. About the new $y^{\prime}$-axis through an angle, $\gamma$

In the new, spherical coordinate system $\left(\theta^{\prime}, \varphi^{\prime}\right)$, the desired ray traces a cone. This ray is given by, $\theta^{\prime}=$ constant $=\Omega$, and with $\varphi^{\prime}$ rotating through 360 degrees as the satellite traces it orbit (i.e. $0<\varphi^{\prime}<2 \pi$ ). The path in celestial coordinates is obtained by transforming back to the original (unrotated) coordinate system. The transformation is:

$$
\begin{gathered}
\varphi=\tan ^{-1}\left[\frac{\sin \theta^{\prime}\left[\sin \psi \cos \gamma \cos \varphi^{\prime}+\cos \psi \sin \varphi^{\prime}\right]+\cos \theta^{\prime} \sin \psi \sin \gamma}{\sin \theta^{\prime}\left[\cos \psi \cos \gamma \cos \varphi^{\prime}-\sin \psi \sin \varphi^{\prime}\right]+\cos \theta^{\prime} \cos \psi \sin \gamma}\right] \\
\theta=\tan ^{-1}\left[\sqrt{\frac{\sin ^{2} \theta^{\prime}\left[\cos ^{2} \gamma \cos ^{2} \varphi^{\prime}+\sin ^{2} \varphi^{\prime}\right]+\cos ^{2} \theta^{\prime} \sin ^{2} \gamma+0.5 \sin \left(2 \theta^{\prime}\right) \sin (2 \gamma) \cos \varphi^{\prime}}{\left[\cos \theta^{\prime} \cos \gamma-\sin \theta^{\prime} \cos \varphi^{\prime} \sin \gamma\right]^{2}}}\right]
\end{gathered}
$$

Substituting Equations $4 \mathrm{~B}$ into Equations 3B, locates the locus of the ray from the antenna bore sight in celestial coordinates. The orbit itself is in a plane perpendicular to $\mathrm{z}^{\prime}$ ( $\mathrm{z}$-axis in the rotated system) and is defined by the rotation angles $\psi$ and $\gamma$. In particular, $\gamma$ is the "inclination" of the plane of the orbit with respect to the $z$-axis (north pole), and $\psi$ determines the equatorial crossing. Because of the choice (definition) made for $\psi$, this occurs on the $\mathrm{y}^{\prime}$-axis. Since, right ascension is measured from the $\mathrm{x}$-axis in celestial coordinates, one has that the right ascension of equatorial crossing of the orbit is: $\alpha=\psi-\pi / 2$.

The case of a sensor with an antenna employing a conical scan is treated in the same manner as described above (for a cross track scan). In the case of a conical scan, a ray from the center of the Earth through the satellite identifies defines "nadir" (the vector $\mathrm{OC}$ in Figure 2B). It also identifies a point in celestial coordinates "above" the satellite that moves as the sensor rotates in its orbit. At each point along this orbit the antenna is 
imagined to do a conical scan about nadir (i.e. about $\mathrm{OC}$ ). If the motion of the satellite can be neglected during each conical scan, then the problem is again identical to the problem above, except that in this case the cone is formed about the vector OC (rather than the normal to orbit, $\mathrm{ON}$, as before). That is, as the antenna does a conical scan with the satellite frozen in space, the reflected ray for the antenna bore sight traces a cone about the nadir, OC, with interior angle $\Omega=2 \theta_{\mathrm{s}}-\theta_{\mathrm{i}}$ as shown in Figure 2B.

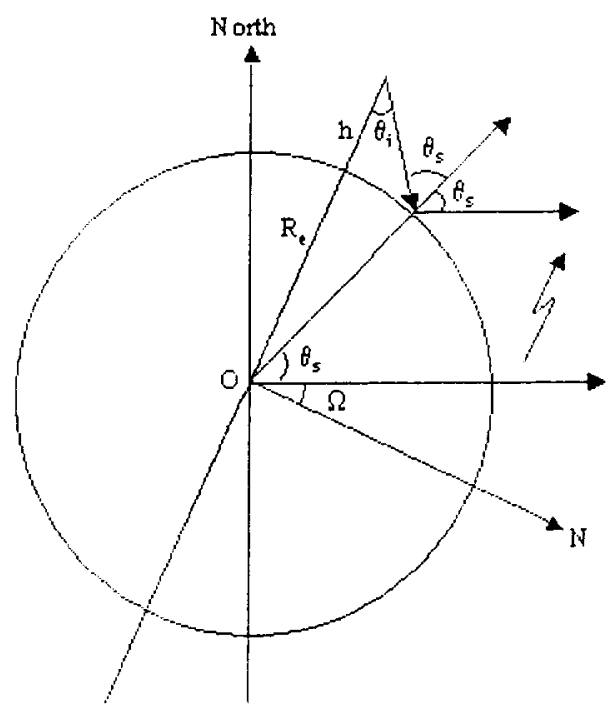

Figure 1B: Geometry for calculation the specular ray at bore sight with cross track scan.

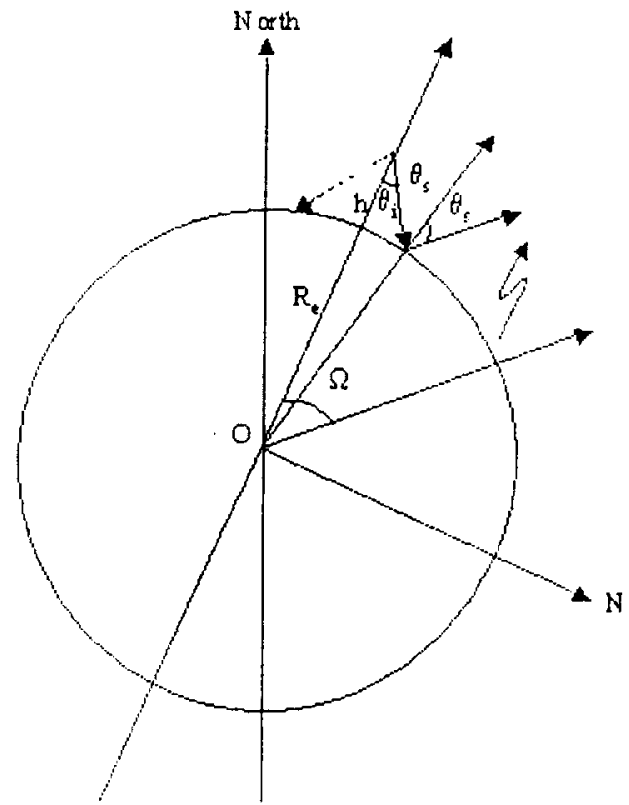

Figure 2B: Geometry for calculation the specular ray at bore sight with conical 


\section{APPENDIX C}

\section{Locating Orbits in Celestial Coordinates}

As illustrated in Appendix B, the idealized orbit can be described by the vector perpendicular to its plane (e.g. ON in Figure 1B) and therefor by two angles that describe this vector. In a celestial coordinate system, by judicious choice, these angles can be declination and right ascension or perhaps their complements. Both geographic and celestial coordinate systems are Earth centered with their axis aligned at the vernal equinox. Latitude and declination are nearly equal. But the relationship between longitude and right ascension is complicated by the rotation of the Earth about its axis and around the Sun.

The relationship between longitude and right ascension is equivalent to the problem of converting from local time at a point on the Earth to sidereal time (time measured in celestial coordinates). This is a problem with nuances caused by fluctuations in the Earth orbit and solved in astronomy [14]. Given a location on the Earth with longitude, $\lambda$, at universal time, $\mathrm{U}_{\mathrm{T}}$, the solution can be written as follows [32]:

$$
\Theta_{\mathrm{L}}=\Theta_{\mathrm{G} 0}+\omega_{\mathrm{E}} \mathrm{U}_{\mathrm{T}}+\lambda \quad 1 \mathrm{C}
$$

where $\Theta_{\mathrm{L}}$ is sidereal time at this point (defines right ascension), $\Theta_{\mathrm{G} 0}$ is sidereal time at Greenwich at midnight of the day on which this calculation is being made (i.e. $0 \mathrm{hr} 0 \mathrm{~min}$ $0 \mathrm{~s}$ ), and $\omega$ is the rate of rotation of the Earth (in the same units as $\mathrm{U}_{\mathrm{T}}$ ). If the units for $\Theta$ and $\lambda$ are degrees and $U_{\mathrm{T}}$ is in minutes, then [32]:

$$
\begin{aligned}
& \omega_{\mathrm{E}}=0.2506846 \mathrm{deg} / \mathrm{min} \\
& \Theta_{\mathrm{G} 0}=100.46062+36000.77 \mathrm{U}_{\mathrm{o}}+0.000388 \mathrm{U}_{0}^{2}-2.6 \times 10^{-8} \mathrm{U}_{0}^{3} \\
& \mathrm{U}_{0}=(\mathrm{JD}-2451544.5) / 36525 \\
& \mathrm{JD}=367 \mathrm{Y}-\mathrm{INT}\{1.75 \mathrm{INT}[(\mathrm{M}+9) / 12]\}+\operatorname{INT}[275 \mathrm{M} / 9]+\mathrm{D}+\mathrm{C}_{0} \\
& \mathrm{C}_{\mathrm{o}}=1721013.5
\end{aligned}
$$

where $\mathrm{Y}$ is the current year (e.g. 2002), $\mathrm{M}$ is the month, $\mathrm{D}$ is the day of the month, and $\mathbb{I N T}[\bullet]$ is the lower nearest integer. Also, JD is the Julian date (number of days at the beginning of the current day since January 1,4713 ) and $\mathrm{U}_{\mathrm{o}}$ is the number of Julian centuries since the reference epoch, January 1, 2000. The expressions above are given in "mean" sidereal time.

When the units in Equation $1 \mathrm{C}$ are in degrees, $\Theta_{\mathrm{L}}$ is equivalent to right ascension. For example, at midnight $U_{T}$ on January 1,2000 , the Greenwich meridian $(\lambda=0)$ is 100.46 degrees east of the mean vernal equinox (the reference for right ascension). Also, 
if the Earth didn't rotate, $\Theta_{\mathrm{L}}$ and $\lambda$ (right ascension and longitude, respectively) would differ by a constant (which could be set to zero).

When an orbit is specified in an Earth centered (geological) coordinate system, Equations $1 \mathrm{C}-2 \mathrm{C}$ can be used to describe it in celestial coordinates. For example, given the equatorial crossing (date, time and longitude) of the orbit (assumed to be in a plane), the expressions above can be used to locate the right ascension of the orbit in celestial coordinates. In the examples presented in this paper (Figures 6-7), it is assumed that the plane of the orbit is frozen in this orientation during one rotation. 

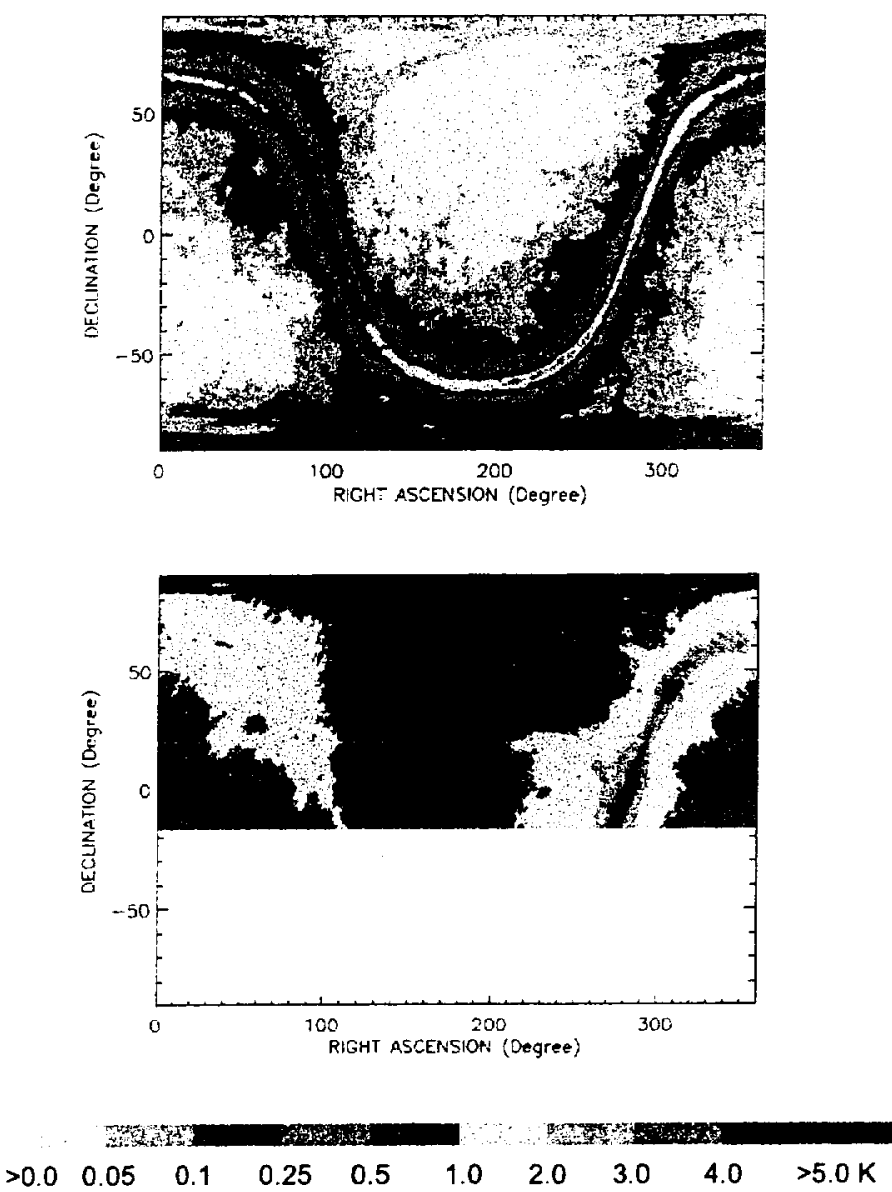

Figure 1: Line emission (top) and continuum background (bottom) as equivalent brightness temperature in $20 \mathrm{MHz}$ bandwidth. 

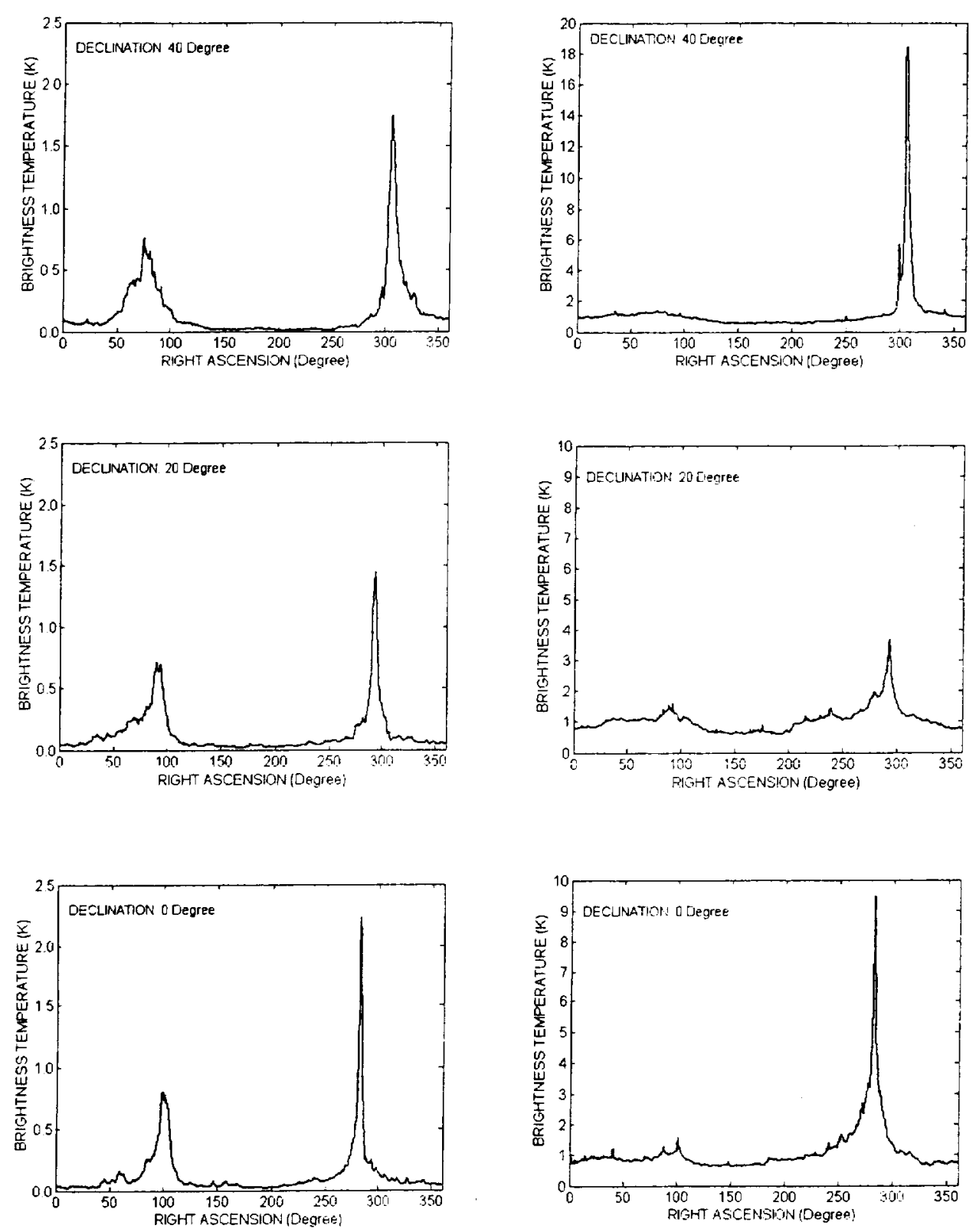

Figure 2: Line emission (left) and continuum background (right) at constant declination of 0, 20 and 40 degrees (bottom to top). Notice that the vertical scale of the upper right panel is $0-20 \mathrm{~K}$. 

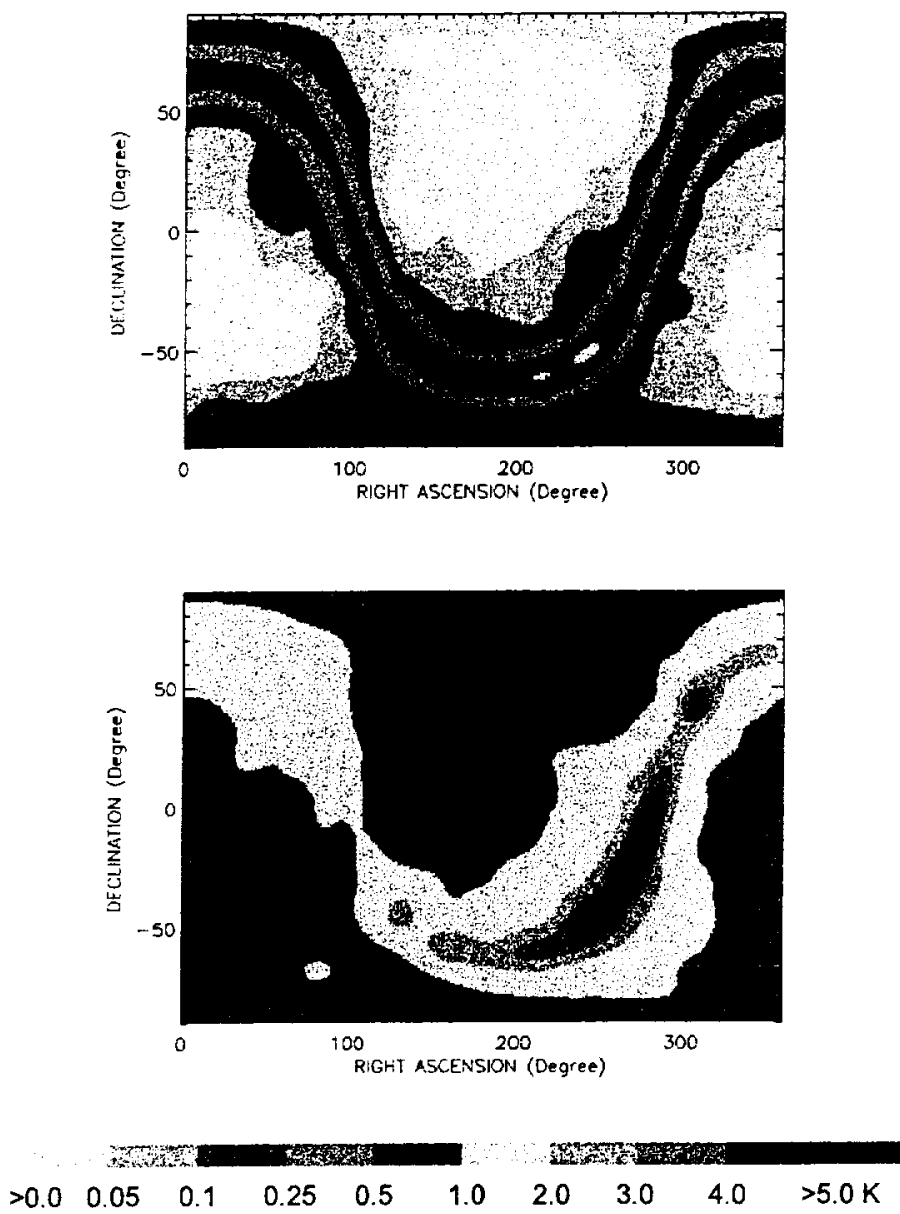

Figure 3: Smoothed data. Line emission (top) and continuum background (bottom) as equivalent brightness temperature in $20 \mathrm{MHz}$ bandwidth as seen by an antenna with a Gaussian beam with a 10 degree beam width (full width at half maximum). 

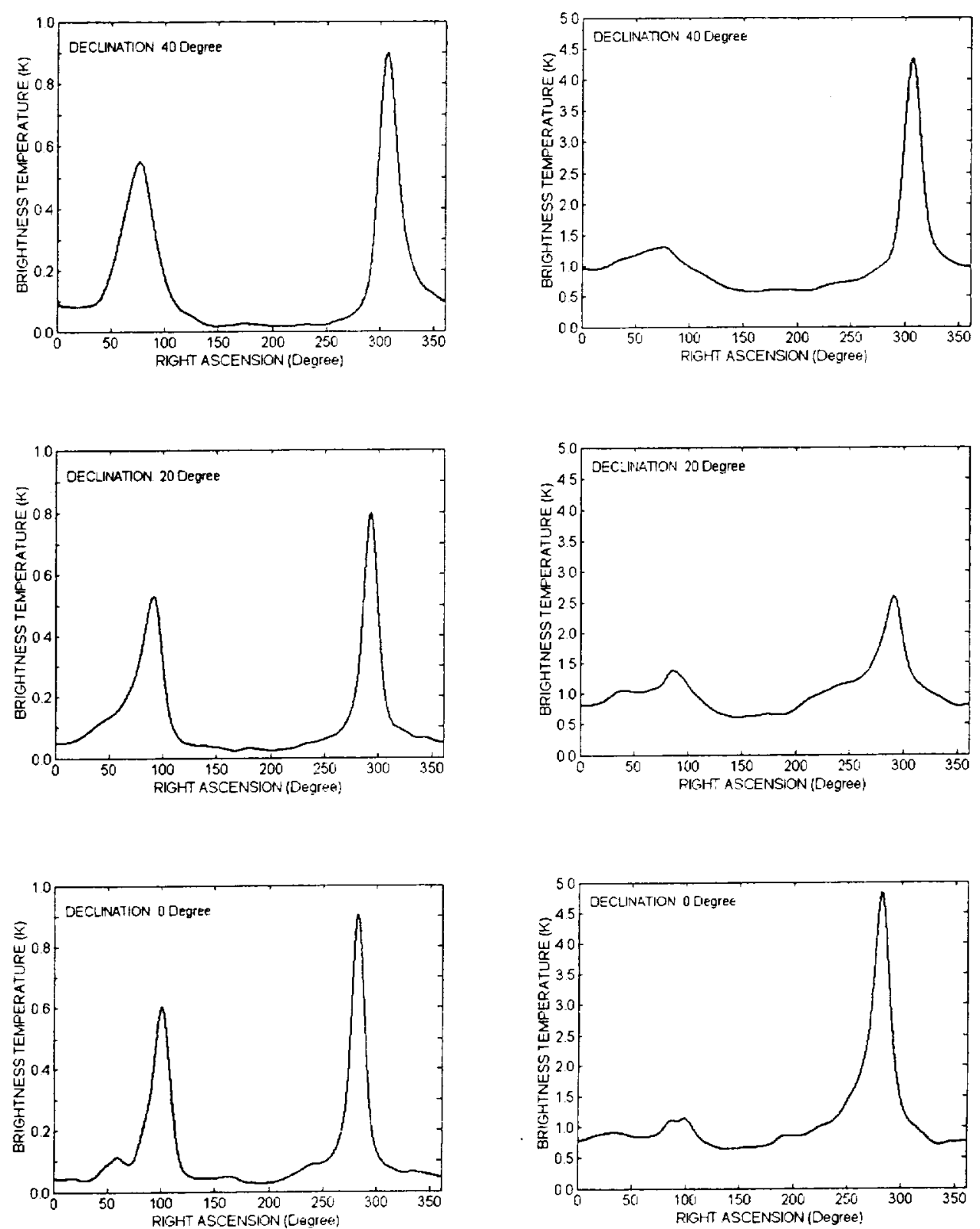

Figure 4: Smoothed data. Line emission (left) and continuum background (right) from Figure 3 but at constant declination of 0,20 and 40 degrees (bottom to top). 

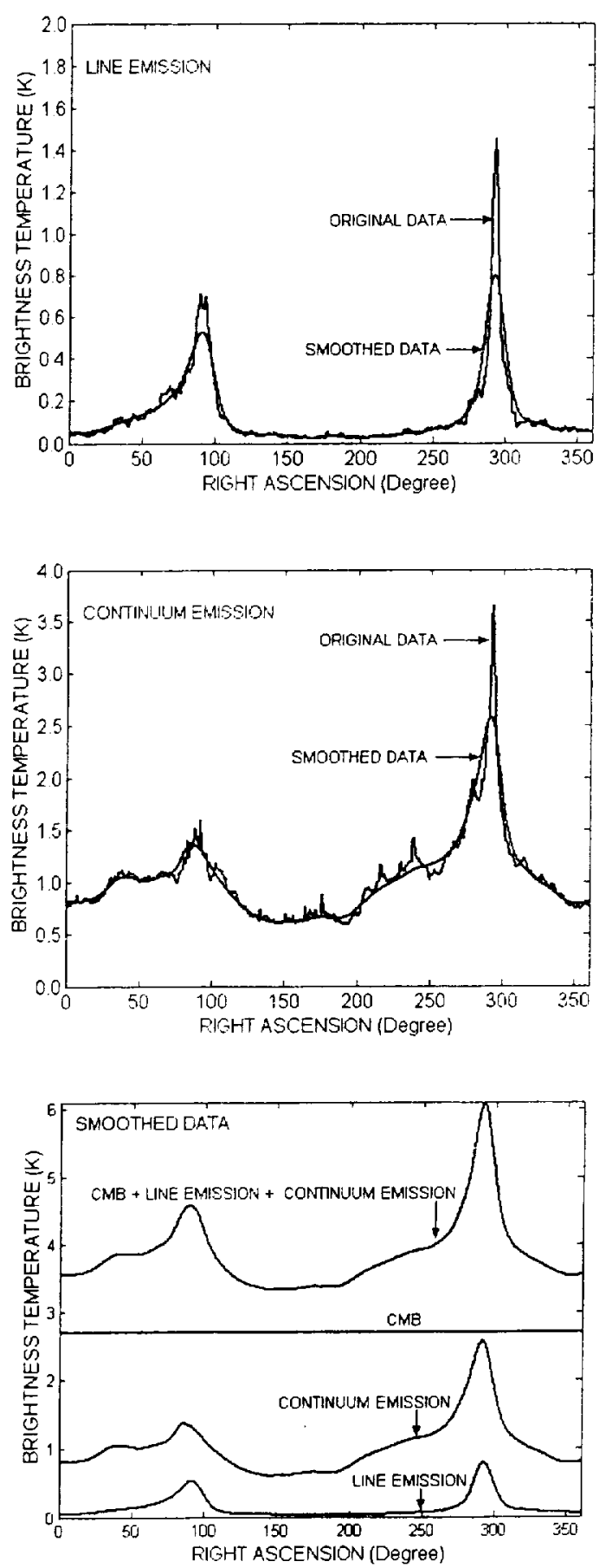

Figure 5: Total background radiation at a constant declination of 20 degrees. The line emission is shown at the top and continuum background in the middle. Each of the components (smoothed) and the CMB is shown at the bottom together with the total. 

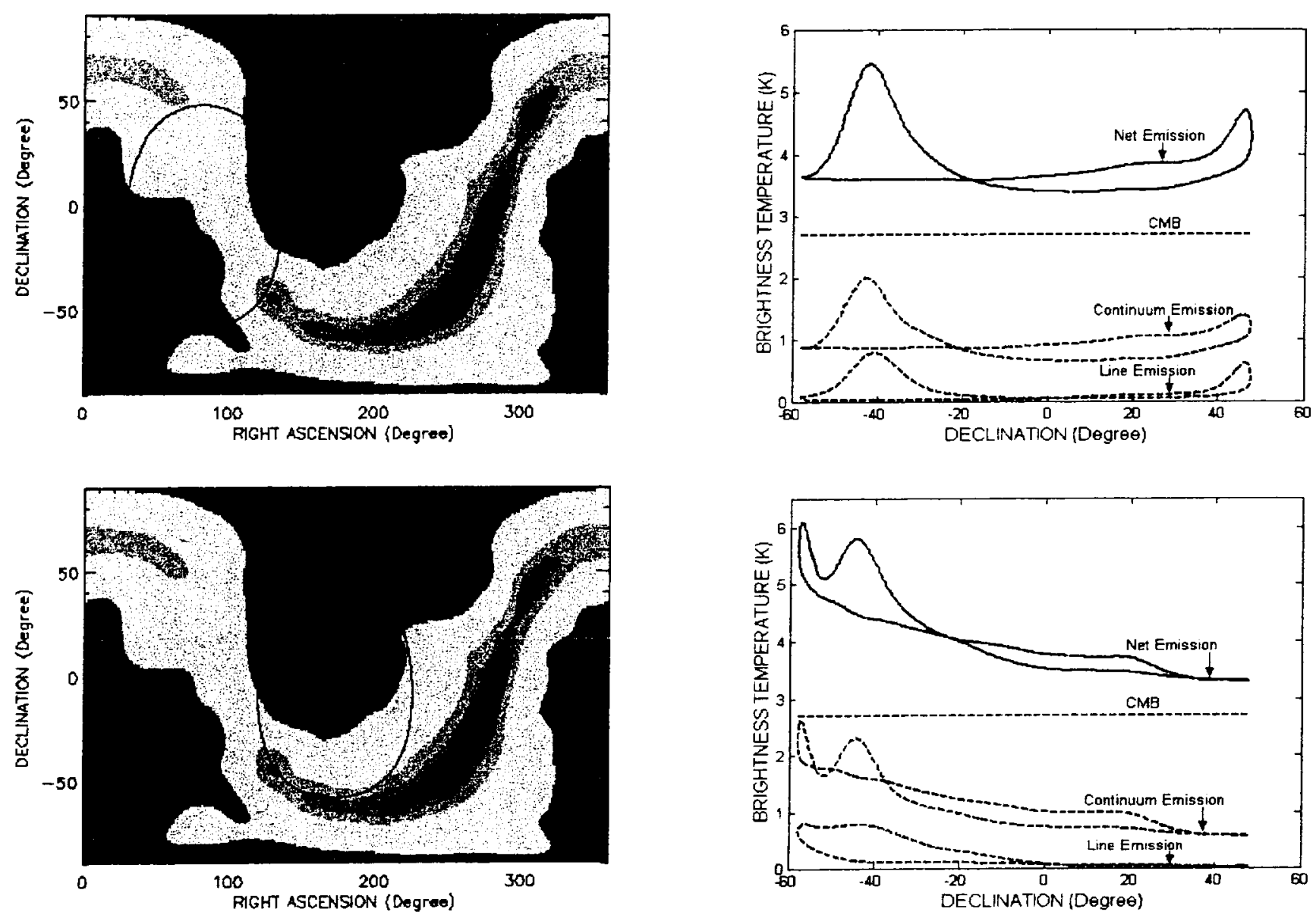

Figure 6: Examples of the background radiation seen by a sensor at look angle 30 degree in a circular, polar orbit with inclination 95 degrees. The two cases are identical except for a change of 90 degrees in the equatorial crossing. The data on the left is the net background (line emission plus continuum) as smoothed with a Gaussian beam with a 10 degree beam width (FWHM). The two panels on the right show the brightness temperature along the locus of the projected beam (solid line) shown on the left. 

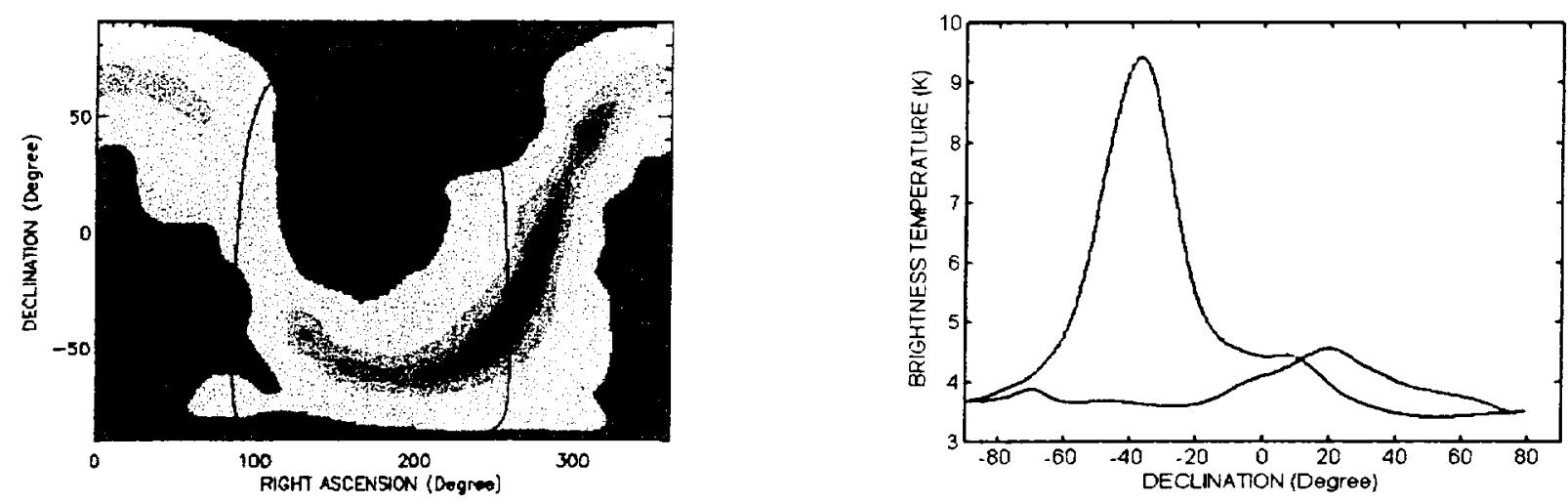

JULY 15, 2002, 00:00:00
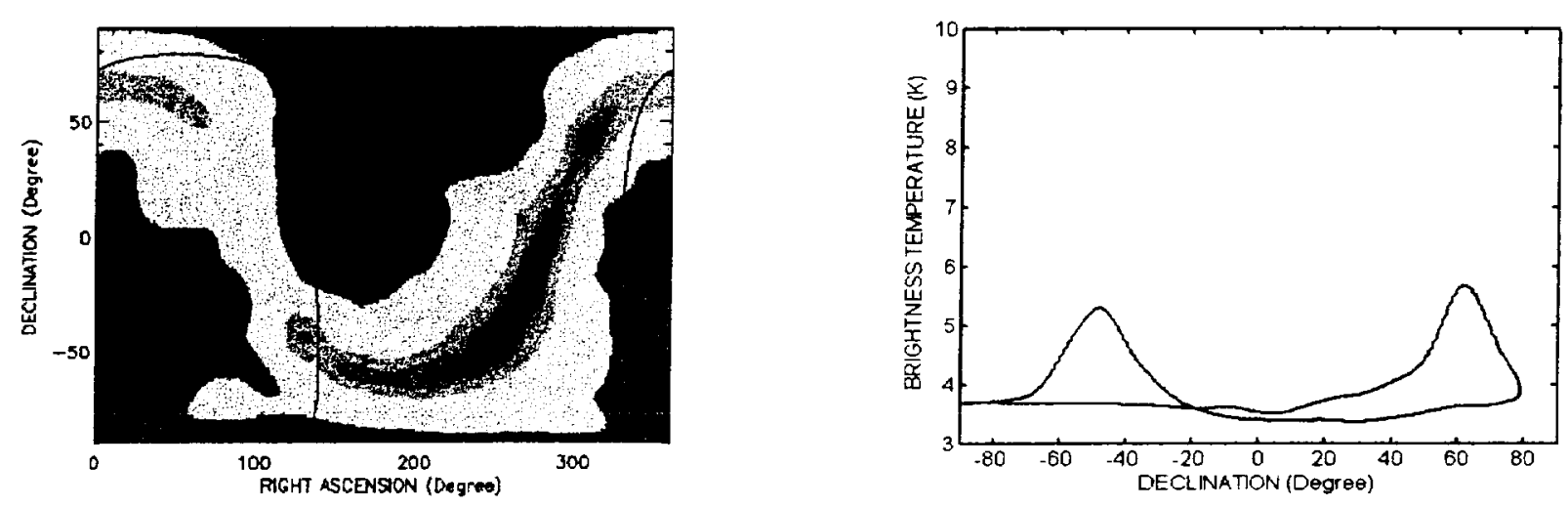

NOVEMBER 15, 2002, 00:00:00
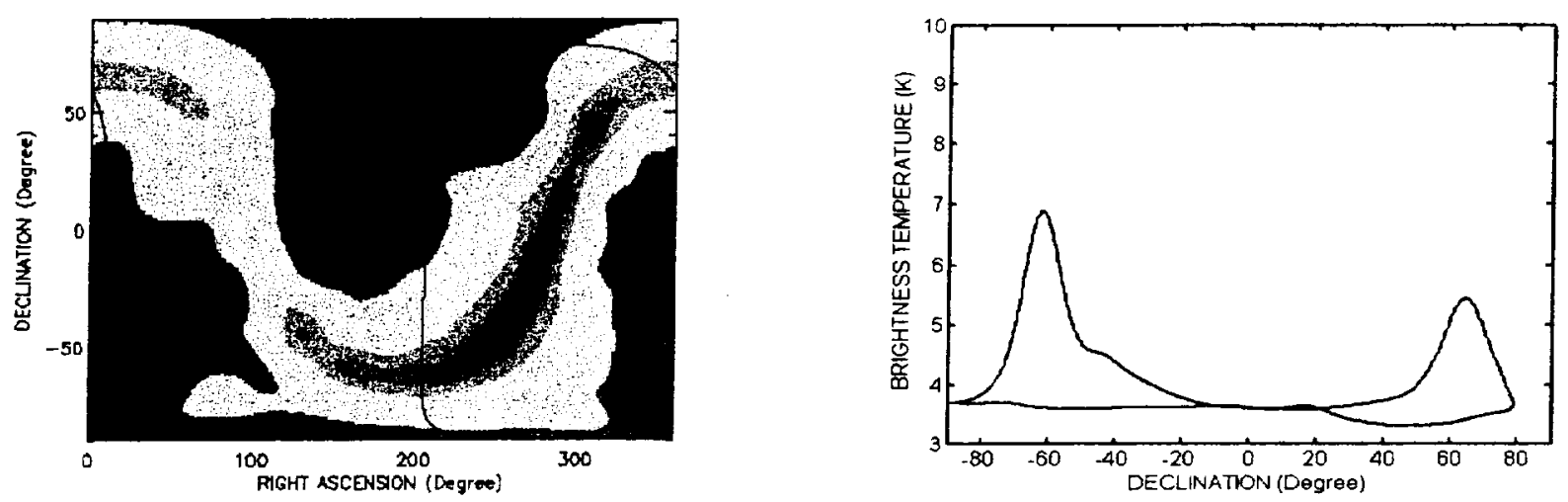

Figure 7: Examples using the HYDROSTAR orbit. The antenna points at 5 degree look angle. The projection of the beam on celestial coordinates is shown on the left. On the right are the values of brightness temperature (the total contribution from line and continuum emission together with the $\mathrm{CMB}$ ). The values are for a sensor with $20 \mathrm{MHz}$ bandwidth and a 10 degree Gaussian beam. 